\title{
Cardiothoracic
}

Transplantation

\section{Antidonor humoral transfer induces transplant arteriosclerosis in aortic and cardiac graft models in rats}

\author{
Bassam Alkhatib, MD, a,b Caroline Freguin-Bouilland, MD, ${ }^{b}$ Pierre Yves Litzler, MD, ${ }^{\text {a }}$ Serge Jacquot, MD, PhD, \\ Françoise Lallemand, ${ }^{\mathrm{b}}$ Jean Paul Henry, ${ }^{\mathrm{b}}$ Christian Thuillez, MD, PhD, ${ }^{\mathrm{b}}$ and Didier Plissonnier, MD, PhD ${ }^{\mathrm{a}, \mathrm{b}}$
}

From the Cardiac and Vascular Surgery Departments, ${ }^{\mathrm{a}}$ INSERM U644, ${ }^{\mathrm{b}}$ and INSERM U512, ${ }^{\mathrm{c}}$ Rouen University Hospital, Rouen, France.

Supported by the Fondation de l'avenir, Paris, France.

Received for publication July 24, 2006; revisions received Oct 16, 2006; accepted for publication Nov 15, 2006.

Address for reprints: Didier Plissonnier, MD, $\mathrm{PhD}$, INSERM U644, Rouen University Hospital, 22 boulevard Gambetta, 76183 Rouen, France (E-mail: didier.plissonnier@churouen.fr)

J Thorac Cardiovasc Surg 2007;133:791-7

$0022-5223 / \$ 32.00$

Copyright () 2007 by The American Association for Thoracic Surgery

doi:10.1016/j.jtcvs.2006.11.015
Objective: The humoral pathway is suggested as playing a key role in transplant arteriosclerosis. The humoral immunity is demonstrated in the present study to induce direct vascular lesion.

Methods: Ten abdominal aortic grafts were performed on 4 groups of rats: Brown Norway (BN) isografts, BN to Lewis (LEW) allografts, and two BN to nude (RNU) grafted groups with and without any humoral transfer. The humoral sera were obtained by skin grafts performed in BN to LEW combination. Lewis anti-BN alloantisera was transferred in nude recipients through intraperitoneal injections. The aortic wall was histologically studied with morphometric analysis on the 21st day. Two additional BN to RNU aortic graft groups were evaluated by immunohistochemistry on days 3 (10 rats) and 10 (10 rats).

Results: In the absence of the humoral transfer, the BN aortic wall implanted in RNU remained intact. The humoral transfer induced a marked intimal proliferation (63 \pm 4 vs $4 \pm 1.1 \mu \mathrm{m} ; P<.001)$ and an adventitial cell infiltration $(5.1 \pm 0.7$ vs $\left.2.8 \pm 0.6 \times 10^{3} \mathrm{c} / \mathrm{mm}^{2}, P<.001\right)$. The medial thickness and the medial cell density were not modified. On day 3 , the remaining endothelial cells were covered by immunoglobulin $\mathrm{G}$ deposits. On day 10 the endothelial cells disappeared completely and intimal proliferation occurred. In an additional cardiac graft group, transplant coronary arteriopathy was evidenced in 7 of the 9 nude recipients that had undergone the humoral transfer.

Conclusion: The transplant arterial occlusive lesion is demonstrated here (1) to be induced by humoral antidonor immunity and (2) to be linked to an adventitial or perivascular inflammation.

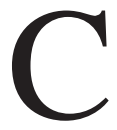
hronic rejection affects the long-term outcome of solid organ transplants. Transplant arteriosclerosis (TA) is the most prominent feature of chronic rejection. TA is defined as an intraluminal obstructive proliferation leading to vessel occlusion. Even if alloimmunity is considered as the main factor, the biologic mechanisms underlying TA have not been clearly established.

The TA histologic lesion is characterized by a diffuse narrowing of arterial lumen including intimal proliferation of smooth muscle cells (SMCs) and fibroblasts, destruction of medial SMCs, and infiltration of adventitial inflammatory cells. ${ }^{1,2}$ In clinical solid organ transplantation, the humoral immunity is critical in the patho- 


\author{
Abbreviations and Acronyms \\ $\mathrm{BN}=$ brown Norway \\ FITC $=$ fluorescein isothiocyanate \\ Ig = immunoglobulin \\ LEW $=$ Lewis \\ $\mathrm{MHC}=$ major histocompatibility complex \\ $\mathrm{RNU}=$ rat nude \\ SMC $=$ smooth muscle cell \\ $\mathrm{TA}=$ transplant arteriosclerosis
}

genesis of TA, particularly alloantibodies directed against donor major histocompatibility complex (MHC) classes I and II. $^{3}$ Anti-HLA class I antibodies have been suggested to induce endothelial and SMC biologic signals ${ }^{4}$ and could initiate an endothelial cell proliferation with regard to vascular lesion development. ${ }^{5}$ These clinical data, together with experimental approaches, demonstrate a close relation between antibody-induced TA and a protective effect of the antiapoptotic gene expression. ${ }^{6}$

In our experience in a rat aortic allograft model, medial smooth muscle destruction appeared to be linked to a humoral immunity. ${ }^{7,8}$ In this model, humoral contributions were at least dependent on MHC-I and involved an apoptosis phenomenon, either in vivo or in vitro, in vascular cell destruction. ${ }^{9}$ Interestingly, adventitial compartment, that is, adventitial inflammatory cells, was demonstrated as a pivotal role in antigen presentation leading to a local antibody production against MHC-I. ${ }^{10}$

In view of this circumstantial evidence, the aim of our study was to demonstrate the capacity of the humoral immunity to induce direct vascular lesions and then to appear as a key for the development of TA.

\section{Materials and Methods}

\section{Animals}

Male rats (200-250 g) from the three inbred strains, NUDE (RNU) NIH-rnu, Brown Norway (BN) RT-1n, and Lewis (LEW) RT-11, were purchased from Iffa-Credo Laboratory (L'Abresle, France). They were used as donor and recipient in an orthotopic aortic graft model and a heterotopic abdominal cardiac graft model. All experiments were conducted according to French legislation for the treatment of animals. Rats were allowed access to food ad libitum.

\section{Experimental Models}

This work was based on humoral transfer experiments. To focus the study on arterial graft intimal lesion, we used experimental models: abdominal aortic allografts as a specific vascular aggression model and abdominal heterotopic cardiac allografts as a solid organ transplant application.

Orthotopic aortic transplantation. The intra-abdominal transplantation was performed under general anesthesia $(20 \mathrm{mg} / \mathrm{kg}$ of ketamine (Imalgene 1000; Merial, Lyon, France). The donor's abdominal aorta $(1.5-\mathrm{cm}$ long) was harvested and flushed with a cold physiologic saline solution and immediately orthotopically transplanted by two end-to-end anastomoses as previously described. ${ }^{11,12}$ The graft ischemic time was 20 to 30 minutes. Aortic clamping ranged from 30 to 40 minutes. No immunosuppressive or anticoagulant treatment was used.

Abdominal heterotopic cardiac transplantation. Intra-abdominal cardiac transplantation was performed under general anesthesia (intramuscular injection of $20 \mathrm{mg} / \mathrm{kg}$ of ketamine. The donor heart was harvested and flushed by a cold physiologic saline solution and immediately heterotopically transplanted, as previously described. ${ }^{13}$ In brief, the donor ascending aorta was anastomosed to the recipient infrarenal abdominal aorta. The donor pulmonary artery was anastomosed to a recipient inferior vena cava. The donor pulmonary veins and caval veins were priory ligated. The graft ischemic time ranged from 40 to 50 minutes. The recipient aortic clamping ranged from 50 to 60 minutes. Neither immunosuppressive nor anticoagulant treatment was used.

\section{Humoral Transfer}

Alloantisera were obtained from LEW after BN skin graft sensitization on an additional rat group. In brief, a $2 \mathrm{~cm}^{2}$-wide full skin patch was obtained from BN and grafted orthotopically onto a LEW rat. Three successive grafts were performed in 10-day intervals. Repeated skin grafts induced serum immunoglobulin $\mathrm{G}(\mathrm{IgG})$ able to recognize vascular cells or splenocytes from the donor's origin $(\mathrm{BN})$ in the fluorescein-activated cell scan as previously described. ${ }^{7}$ One million BN splenocytes were incubated for 30 minutes at $4^{\circ} \mathrm{C}$ with $10 \mu \mathrm{L}$ of alloantisera IgG diluted from $1: 1$ to 1:1000. Cells, washed twice in phosphate-buffered saline solutionfetal calf serum, were incubated with fluorescein isothiocyanate (FITC)-conjugated mouse anti-rat Ig kappa chain. The alloantiserum used for transfer was 1:50 diluted. The aortic allografted recipient rats were intraperitoneally injected with $1.5 \mathrm{~mL}$ alloantisera 3 days before the aortic transplantation, the day of the transplantation, and 2 and 5 days after the transplantation. Cardiac allografted recipient rats were intraperitoneally injected with $1.5 \mathrm{~mL}$ alloantisera the day of the transplantation and $2,5,7$, and 9 days after the transplantation.

\section{Experimental Design}

The orthotopic aortic transplantation was performed in 4 groups of 10 rats put to death on the 21st day: a $\mathrm{BN}$ to $\mathrm{BN}$ isografted group (group 1), a BN to LEW allografted group (group 2), a BN to RNU grafted group without any humoral transfer (group 3), and a $\mathrm{BN}$ to RNU grafted group with a humoral transfer (group 4).

To study early lesions, we performed 2 additional $\mathrm{BN}$ to RNU aortic graft groups with and without humoral transfer $(n=10$, respectively). In these 2 groups, rats were put to death on day 3 $(n=5)$ and on day $10(n=5)$. For both of those experiments, the humoral transfer was performed 3 days before and the day of the aortic transplantation.

The heterotopic abdominal cardiac transplantation was performed in 2 groups of 10 rats put to death on day 21: a BN to RNU cardiac graft group without any humoral transfer (group A) and a $\mathrm{BN}$ to RNU cardiac graft group with a humoral transfer (group B).

All rats were put to death with an overdose of pentobarbital $(50 \mathrm{mg} / \mathrm{kg})$. Cardiac and aortic grafts were rapidly dissected and removed. 


\section{Histology}

A histologic examination was performed in all groups. For this purpose, after harvesting, aortas and hearts were flushed with a physiologic saline solution. One half of each graft was postfixed in $4 \%$ formalin for 24 hours. The tissue was embedded in paraffin, and transversal sections of $5 \mu \mathrm{m}$ were performed to allow light microscopic examination. Slides were stained with orcein, sirius red, and hematoxylin, for elastin, collagen, and nuclei, respectively. Each slide was examined for qualitative and quantitative evaluation.

The other half of the graft was snap-frozen immediately in isopentane precooled by liquid nitrogen after filling with a fixative solution (Tissue TEK; Miles Laboratories, Elkart, Ind). The cryopreserved half of the transplant was stored at $-80^{\circ} \mathrm{C}$ for cryostat section $(5-\mu \mathrm{m}$ thick). Immunohistochemical staining was performed with streptavidine peroxidase technique (Amersham, Buckinghamshire, United Kingdom) to reveal IgG deposits by a monoclonal antibody against rat IgG (Biotinylated Anti-rat IgG; Victor Laboratories, Burlingam, Calif).

\section{In Situ Detection of DNA Fragmentation}

DNA fragmentation was detected in situ by the terminal deoxynucleotidyl transferase-mediated dUTP nick end labeling (TUNEL) method (In situ Cell Death Detection Kit; Boehringer, Mannheim, Germany). DNA fragmentation was evaluated after a 1-hour incubation at $37^{\circ} \mathrm{C}$ with terminal deoxynucleotidyl transferase FITCconjugated dUTP.

Slides were examined with a Leica fluorescence microscope (Leica Microsystems GmbH, Wetzlar, Germany).

\section{Morphometric Analysis}

A semiautomatic image analysis was used to quantify histologic changes on a formalin-fixed section for the aortic grafts. The image system included the following: a light microscope (Nikon E600 microscope; Nikon, Tokyo, Japan), a color video camera (Spot Software v2.21 Camera, Diagnostic Instruments, Inc, Sterling Heights, Mich), an image analysis processor (Software Image Pro-Plus 4.1 for Windows 95), and a personal computer connected to the processor that permitted the automatic analysis of all morphologic transformations and sequential storage of the results. For each stain, measurements were performed on aortic intima, media, and adventitia tunicae and on coronary arteries. Two programs were used in this study. The first program was used to analyze the elastin structure of the aortic wall. The elastin contents permitted the determination of the intimal and medial thickness micrometers. The second program was used to analyze the nuclear contents of the graft wall. The mean cell density (number of nuclei per square millimeter of the aortic wall) was measured in adventitia (adventitial cell density: cells per square millimeter) and media (medial cell density: cells per square millimeter).

\section{Statistical Analysis}

In the aortic graft model, the results of the morphometric study are expressed as mean $\pm \mathrm{SEM}$. The Student $t$ test was performed to analyze the differences in morphometric measurements. Analysis of variance was used as a single factor for the different parameters (Systat program version 8.0-1998; Systat Software, Inc, San Jose, Calif).
In the cardiac graft model, the study was focused on coronary arteries, particularly intimal lesions. Each heart was studied on four 1-mm-separated levels. All slides were carefully examined. The detection of at least one coronary intimal proliferative lesion assigned the entire cardiac graft submitted to the pathologic process. The coronary lesions affecting the cardiac grafts were counted in the $2 \mathrm{BN}$ to RNU groups. Then we used the $\chi^{2}$ Pearson test.

\section{Results}

No sign of rejection was observed in the isografted aorta: there was neither intimal proliferation nor inflammatory adventitial infiltration; the medial thickness was respected (Figure 1, A).

When allografted in LEW recipients, BN aortas underwent a severe rejection process (Figure $1, B$ ) as compared with BN/BN isografts: the intimal thickness and the intima/ media ratio increased (Table $1 ; P<.001$ ), the medial thickness and the medial smooth cell density decreased (Table $1 ; P<.001$ ), and the adventitial cell density increased (Table $1 ; P<.001$ ).

In the absence of the humoral transfer, the aspects of the three compartments of BN aortas grafted in RNU recipients (Figure 1,C) were not significantly different from those of the isografted aorta: intimal thickness $(P=1)$, medial thickness $(P=.8)$, intima/media ratio $(P=1)$, medial cell density $(P=.15)$, and adventitial cell density $(P=.71)$.

The humoral transfer induced severe lesions in the BN aorta grafted in RNU recipients (Figure 1, D). When compared with RNU recipients alone, the humoral transfer induced intimal thickening ( $63 \pm 4$ vs $4 \pm 1.1 \mu \mathrm{m}, P<.001$ ), mostly composed of a myofibroblastic cell proliferation, covered by an endothelial cell lining. The endothelial layer was not characterized in this study. The media seemed to be unaltered by the humoral transfer ( $71 \pm 9$ vs $81 \pm 12 \mu \mathrm{m}$; $P=.15)$. The intima/media ratio increased in relation to the intimal thickness $(81 \pm 5$ vs $4.5 \pm 1.2 ; P<.001)$. The medial cell density was similar in the 2 groups $(2.7 \pm 0.4$ vs $\left.3.3 \pm 0.7 \times 10^{3} \mathrm{cell} / \mathrm{mm}^{2} ; P=.34\right)$. Unexpectedly, the humoral transfer increased the adventitial cell density in RNU recipients $\left(5.1 \pm 0.7\right.$ vs $2.8 \pm 0.6 \times 10^{3} \mathrm{cell} / \mathrm{mm}^{2} ; P$ $=.026$ ).

However, the aortic aggression induced by humoral transfer in RNU recipient was moderate when compared with LEW recipient allografts. The intimal thickness was lower than in LEW recipient allografts $(63 \pm 4$ vs $299 \pm 54 \mu \mathrm{m} ; P<$ .001); the medial thickness remained higher than in LEW recipient allografts $(71 \pm 9$ vs $45.2 \pm 4 \mu \mathrm{m} ; P<.001)$, and the ratio intima/media was lower $(81 \pm 5$ vs $450 \pm 67 ; P<$ $.001)$; the medial SMC density was similar in the 2 groups $\left(2.7 \pm 0.4\right.$ vs $\left.2.3 \pm 1 \times 10^{3} \mathrm{cell} / \mathrm{mm}^{2} P=.67\right)$; the adventitial cell density was lower in the humoral transfer group than in the LEW recipient $(5.1 \pm 0.7$ vs $9.5 \pm 3.6 \times$ $\left.10^{3} \mathrm{cell} / \mathrm{mm}^{2} P=.001\right)$. 

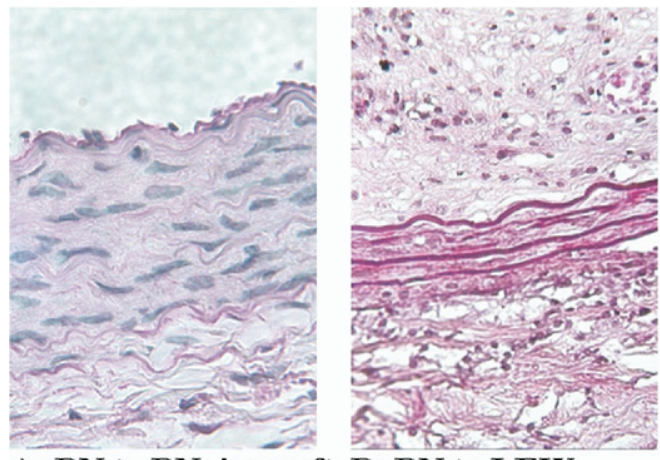

$A$ : BN to BN isograft $B$ : $B N$ to $\mathrm{LEW}$

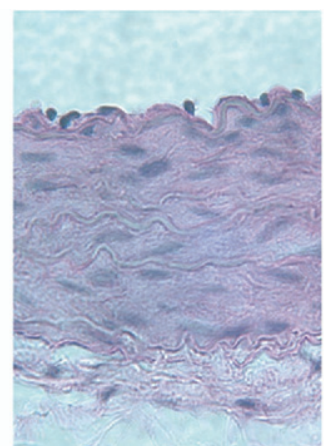

C: BN to RNU without humoral transfer

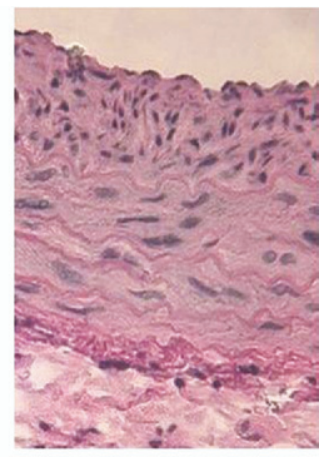

D: BN to RNU with humoral transfer

Figure 1. Histologic aspects of BN aortic graft on the 21st day: effect of the humoral (anti BN) transfer in RNU recipient (hematein). The isografted aorta was devoid of any rejection sign: intimal and medial layers were respected, and inflammatory adventitial infiltration was rare (A). In contrast, BN to LEW allografted aorta exhibited a marked obstructive intimal proliferation, a destruction of the elastic laminae, and the disappearance of SMC in the media; adventitial cell infiltration was prominent (B). In the RNU recipient without humoral transfer, the BN aortic wall was similar to the isografted aorta (C). Humoral transfer induced intimal thickening. Interestingly enough, medial layers seemed to be respected and adventitial inflammatory infiltration occurred (D). BN, brown Norway; LEW, Lewis; $R N U$, rat nude; SMC, smooth muscle cell.

To approach the intimal lesion induced by the humoral transfer in nude recipients, we performed 2 additional aortic grafted groups in RNU recipients and submitted them to immunohistologic analysis. When the humoral transfer occurred, an immunohistologic study revealed the presence of LEW anti-BN antibodies ( $\operatorname{IgG})$ evidenced on remaining endothelial lining when sacrificed on day 3 (Figure 2, F). In the absence of the humoral transfer, endothelial cell lining was free of any IgG deposits. Later, on day 10, the endothelial cells disappeared and an intimal proliferation occurred within the humoral transfer group (Figure 2, $G$ ). In the absence of the humoral transfer, the endothelial cells remained intact (Figure $2, C$ ). When focusing on endothelial cells, studies revealed several signs in favor of an apoptosis phenomenon. In the humoral transferred group, hematoxylin coloration evidenced nuclear condensation on day 3 (Figure 2, E). Later, on day 21, TUNEL was positively evidenced in endothelial lining and intimal thickness (Figure 2, $H$ ).
Beside the aortic allografts, 2 groups of cardiac allografts were performed in RNU recipients to test the capacity of the humoral transfer to induce a coronary lesion. Sacrifices on day 21 showed prominent obliterative lesions in coronary arteries in 7 of the 9 transplanted hearts of the humoraltransferred group (Figure 3). The obliterative lesions were essentially composed of myofibroblastic cell proliferation and tended to a complete occlusion. Coronary lesions were closely associated with adventitial perivascular cell inflammatory infiltrates. The histologic examination failed to evidence any change in the cardiac parenchymal tissue. In the absence of the humoral transfer, the coronary arteries and cardiac parenchyma were intact.

\section{Discussion}

The main result of this study was the demonstration that humoral immunity was able in vivo to induce vascular endothelial cell destruction and intimal thickening to

TABLE 1. Quantitative morphologic changes in intima, media, and adventitia occurring in BN aortic graft on the 21st day: effect of the humoral (anti-BN) transfer in nude rat

\begin{tabular}{lcccc}
\hline & BN recipient & LEW recipient & $\begin{array}{c}\text { RNU recipient without } \\
\text { humoral transfer }\end{array}$ & $\begin{array}{c}\text { RNU recipient with } \\
\text { humoral transfer }\end{array}$ \\
\hline Intimal thickness $(\mu \mathrm{m})$ & $3.4 \pm 1.2$ & $299 \pm 54$ & $4 \pm 1.1$ & $63 \pm 4$ \\
Medial thickness $(\mu \mathrm{m})$ & $85.3 \pm 6.6$ & $45.2 \pm 4$ & $81 \pm 12$ & $71 \pm 9$ \\
Intima/media ratio & $3.9 \pm 1.3$ & $450 \pm 67$ & $4.5 \pm 1.2$ & $81 \pm 5$ \\
Medial cell density $\left(\times 10^{3}\right.$ cell/ $\left./ \mathrm{mm}^{2}\right)$ & $3.7 \pm 0.5$ & $2.3 \pm 1$ & $3.3 \pm 0.7$ & $2.7 \pm 0.4$ \\
Adventitial cell Density $\left(\times 10^{3} \mathrm{cell}^{2} \mathrm{~mm}^{2}\right)$ & $3.7 \pm 0.8$ & $9.5 \pm 3.6$ & $2.8 \pm 0.6$ & $5.1 \pm 0.7$ \\
\hline
\end{tabular}

$B N$, brown Norway; LEW, Lewis; $R N U$, rat nude. 


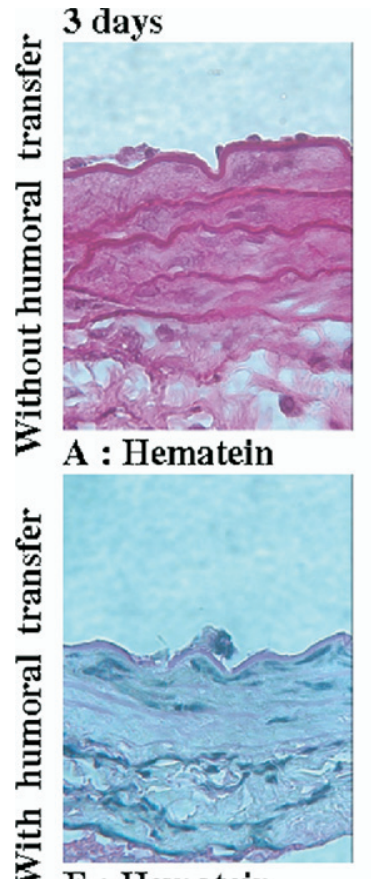

E : Hematein

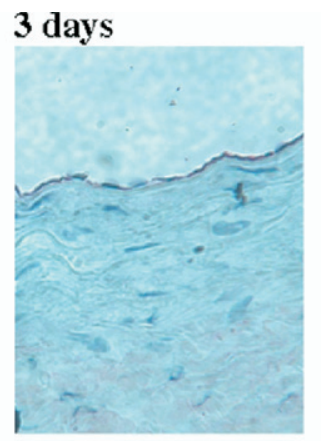

B : anti-IgG

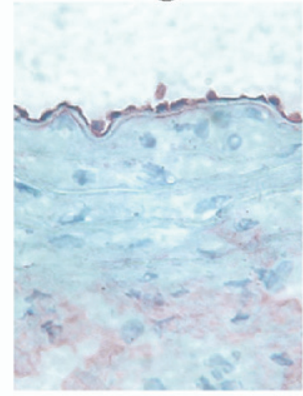

F : anti-IgG

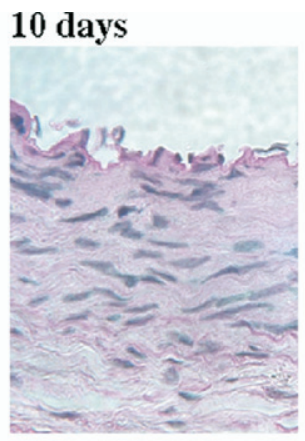

\section{C : Hematein}

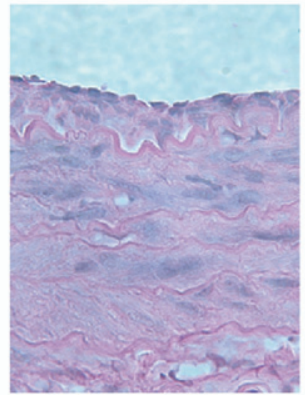

G : Hematein

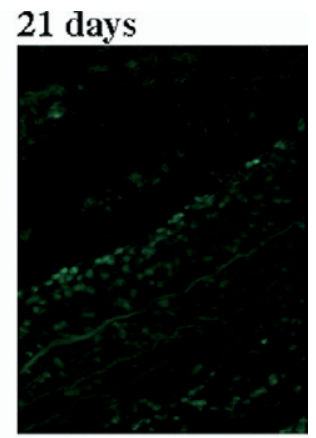

D : Tunel

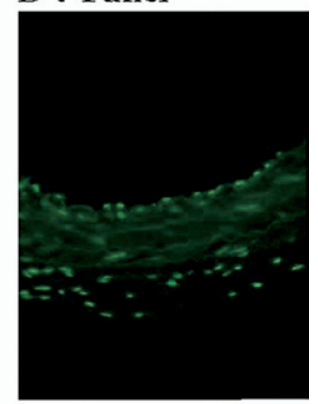

H : Tunel

Figure 2. Immunohistological aspects of BN aortic graft on the third, 10th, and 21st days: effect of the humoral (anti-BN) transfer in RNU recipient. (Hematein staining; IgG deposits were revealed by biotinylated anti-rat IgG monoclonal antibodies; TUNEL staining). This figure is focused on the intima and luminal part of the media. In the absence of the humoral transfer, the intima was intact on days 3 and 10 ( $A$ and $C$, hematein). Endothelial cell lining was devoid of any IgG staining on day 3 (B). On day 21, TUNEL staining revealed a very slight apoptosis affecting rare endothelial cells (D). TUNEL was negative in medial and adventitial layers. In contrast, the humoral transfer induced intimal destruction on day 3 with the disappearance of endothelial cells (E: hematein). The presence of LEW anti-BN antibodies (IgG) deposits was revealed on the remaining endothelial cells (F). On day 10 an intimal thickening occurred (G). On day 21, TUNEL staining revealed markedly an apoptosis in the intimal proliferation and adventitial layers (H). BN, brown Norway; $R N U$, rat nude; IgG, immunoglobulin G; LEW, Lewis.

develop as a response to immune arterial wall aggression. This result was obtained in rat models of aortic and cardiac graft. When BN aortas were transplanted in RNU recipients in the absence of humoral transfer, aortic grafts were devoid of any rejection process. Endothelial cell lining and the media were respected. The adventitia was not invaded by inflammatory cells, arguing for a true tolerance to aortic graft by the RNU strain. The humoral anti-BN transfer had a striking effect on the arterial wall as confirmed by the quantitative and qualitative histologic findings. This is consistent with a direct effect of antibodies on the arterial wall and, in particular, on endothelial cells. Intimal thickening developed as a response to immune endothelial aggression. Early observations performed on BN aortic graft after humoral (LEW anti-BN) transfer in RNU recipient revealed the presence of $\mathrm{IgG}$ deposits on the remaining endothelial cells on day 3. A few days later (day 10) the endothelial cells disappeared completely and the intimal thickening devel- oped. The same process was evidenced on cardiac grafts performed in nude recipients. Without any humoral anti-BN antibody transfer, cardiac allografts were devoid of any sign of rejection: neither coronary lesion nor parenchymal inflammatory infiltration was observed in 21 days. The humoral transfer led to a marked intimal proliferation, occurring in 7 of the 9 cardiac grafted rats.

The rat aortic graft model has been used for several years to study the arterial lesions occurring in chronic rejection in allografts. In our experiment in the model of LEW recipient of BN aorta, medial smooth muscle destruction appeared to be linked to humoral immunity. ${ }^{7,9}$ Moreover, the skin graft presensitization increased the humoral immunity, medial SMC destruction, and accelerated intimal proliferation. ${ }^{8}$ Alloantibodies were eluted from aortic allografts and were characterized mostly as anti-MHC-I molecules. The humoral immunity was demonstrated to be capable of aortic wall cell destruction, particularly in vitro, through an apoptotic phenomenon. ${ }^{9}$ Furthermore, the present study 


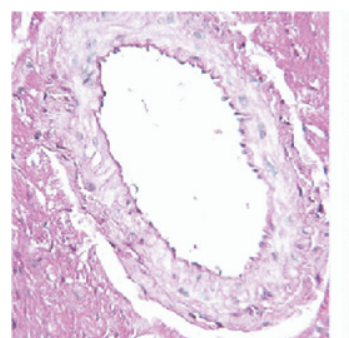

A

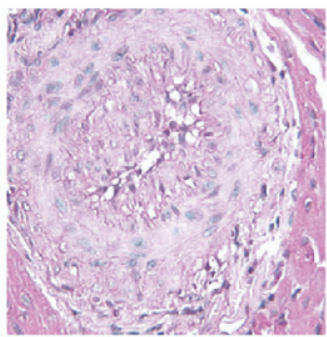

B

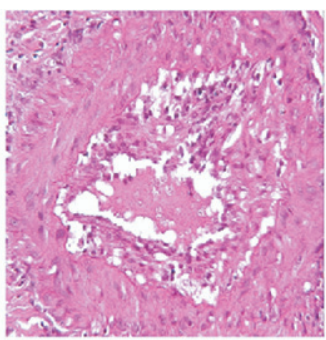

C

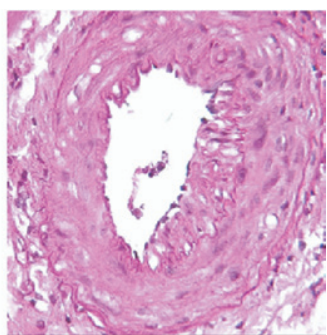

D

Figure 3. Histologic aspects of BN cardiac graft on the 21st day: effect of the humoral (anti-BN) transfer on coronary artery in RNU recipient (hematein). In the absence of the humoral transfer, no coronary lesion was found in any of the $9 \mathrm{BN}$ cardiac grafts performed in RNU recipient (A). In contrast, the humoral transfer induced coronary lesions in 7 of the 9 BN cardiac grafts performed in RNU recipient. Transplant coronary lesions caused by the humoral transfer were characterized by a massive intimal proliferation leading to a coronary artery occlusion (B, C, and D). Intimal lesions were closely associated with perivascular adventitial inflammatory cell infiltrates (B and C). BN, brown Norway; $R N U$, rat nude.

showed a direct in vivo vascular wall aggression by humoral immunity. On day 21, TUNEL staining was in favor of an apoptotic phenomenon occurring on luminal cells. Our results are in agreement with an experimental cardiac graft model submitted to humoral transfer. ${ }^{6}$ These authors showed that humoral transfer can induce coronary lesions in the cardiac graft in mice and that this is linked to an apoptotic phenomenon. In fact, antiapoptotic genes like Bcl-xl or oxygenase-1 promoted protective effect on coronary lesions. ${ }^{6}$ In the present study, the humoral phenotype or molecular approach was not determined before the transfer in RNU recipients. We previously demonstrated the role of MHC-I involved in the vascular rejection process during the aortic allograft in rats. ${ }^{9}$ Clear links between coronary proliferation and indirect allorecognition of donor MHC-I have been reported in cardiac transplantation during experimental $^{14}$ or clinical studies. ${ }^{5}$ Moreover, use of an antiMHC-I antibody has been found to promote endothelial cell proliferation ${ }^{5}$ and activate signal transduction pathways in SMCs. $^{4}$

Interestingly, in our study humoral transfer was accompanied by an adventitial cell infiltration. New concepts evidenced adventitial lymphoids as the source of humoral alloimmune response against donor MHC-I in chronic vascular rejection. ${ }^{10}$ A key role was attributed to adventitial cell infiltrates for local humoral production. Our results are to be considered with the previous results. In nude recipients without humoral transfer, $\mathrm{BN}$ endothelial cells were intact and the adventitial layer was devoid of any inflammatory cells. When the humoral transfer was performed first, endothelial cells were covered by $\operatorname{IgG}$ on day 3 , then destroyed on day 5 by direct IgG aggression. Intimal thickening developed as soon as day 10 in response to endothelial aggression. Second, adventitial inflammatory cell infiltration was prominent on day 21, arguing for local immune response to antigen presentation.

The same process was observed in RNU cardiac graft recipients: intimal lesions were prominent on day 21 and closely associated with perivascular space infiltration by inflammatory cells. The parenchymal tissue was free from any inflammatory infiltration or fibrosis. In the absence of the humoral transfer, coronary arteries remained intact. The humoral transfer induced the formation of obstructive coronary lesion, closely with adventitial perivascular inflammatory infiltration.

In conclusion, this model could be relevant to the study of TA. We have demonstrated here, first, a key role for antidonor antibodies in the induction of transplant arterial occlusive lesion and, second, a clear link between transplant intimal lesion and adventitial or perivascular inflammation.

\section{References}

1. Hillebrands JL, Klatter FA, van den Hurk BMH, Popa ER, Nieuwenhuis P, Rozing J. Origin of neointimal endothelium and $\alpha$-actinpositive smooth muscle cells in transplant arteriosclerosis. $J$ Clin Invest. 2001;107:1411-22.

2. Hillebrands JL, Onuta G, Rozing J. Role of progenitor cells in transplant arteriosclerosis. Trends Cardiovasc Med. 2005;15:1-8.

3. Rifle G, Mousson C, Martin L, Guignier F, Hajji K. Donor-specific antibodies in allograft rejection: clinical and experimental data. Transplantation. 2005;79:S14-8.

4. Bian H, Reed EF. Alloantibody-mediated class I signal transduction in endothelial cells and smooth muscle cells: enhancement by IFN- $\gamma$ and TNF- $\alpha$. J Immunol. 1999;163:1010-8.

5. Jin YP, Jindra PT, Gong KW, Lepin EJ, Reed EF. Anti-HLA class I antibodies activate endothelial cells and promote chronic rejection. Transplantation. 2005;79:S19-21.

6. Hancock WW, Buelow R, Sayegh MH, Turka LA. Antibody-induced transplant arteriosclerosis is prevented by graft expression of antioxidant and anti-apoptotic genes. Nat Med. 1998;4:1392-6. 
7. Plissonnier D, Nochy D, Poncet P, Mandet C, Hinglais N, Bariety J, et al. Sequential immunological targeting of chronic experimental arterial allograft. Transplantation. 1995;60:414-24.

8. Kolb F, Heudes D, Mandet C, Plissonnier D, Osborne-Pellegrin M, Bariety J, et al. Presensitization accelerates allograft arteriosclerosis. Transplantation. 1996;62:1401-10.

9. Plissonnier D, Henaff M, Poncet P, Paris E, Tron F, Thuillez C, et al. Involvement of antibody-dependent apoptosis in graft rejection. Transplantation. 2000;69:2601-8.

10. Thaunat O, Field AC, Dai J, Louedec L, Patey N, Bloch MF, et al. Lymphoid neogenesis in chronic rejection: evidence for a local humoral alloimmune response. Proc Natl Acad Sci U S A. 2005;102:14723-8.

11. Plissonnier D, Levy BL, Salzmann JL, Nochy D, Watelet J, Michel JB. Allograft-induced arterial wall injury and response in normo- tensive and spontaneously hypertensive rats. Arterioscler Thromb. 1991;11:1690-9.

12. Plissonnier D, Amichot G, Legagneux J, Duriez M, Gentric D, Michel JB. Additive and synergistic effects of a low-molecular-weight heparinlike molecule and low doses of cyclosporin in preventing arterial graft rejection in rats. Arterioscler Thromb. 1993;13:112-9.

13. Alkhatib B, Freguin-Bouilland C, Lallemand F, Henry JP, Litzler PY, Marie JP, et al. Low molecular weight fucan prevents transplant coronaropathy in rat cardiac allograft model. Transpl Immunol. 2006; 16:14-9.

14. Lee RS, Yamada K, Houser SL, Womer KL, Maloney ME, Rose HS, et al. Indirect recognition of allopeptides promotes the development of cardiac allograft vasculopathy. Proc Natl Acad Sci U S A. 2001;98: 3276-81. 